OPEN ACCESS

Edited by:

Tzvi Dwolatzky,

Technion Israel Institute of

Technology, Israel

Reviewed by:

Melissa Orlandin Premaor,

Federal University of Minas

Gerais, Brazil

Stefano Pagano,

University of Perugia, Italy

${ }^{*}$ Correspondence:

Mingxiang Yu

yu.mingxiang@zs-hospital.sh.cn

Xinhua Qu

xinhua_qu@126.com

†These authors have contributed equally to this work

Specialty section:

This article was submitted to

Bone Research

a section of the journal

Frontiers in Endocrinology

Received: 28 February 2020

Accepted: 23 June 2020

Published: 30 July 2020

Citation:

Yang Y, Liu G, Zhang Y, Xu G, Yi X

Liang J, Zhao C, Liang J, Ma C, Ye Y,

Yu M and Qu X (2020) Linear and

Non-linear Correlations Between

Serum Phosphate Level and Bone

Mineral Density in Type 2 Diabetes.

Front. Endocrinol. 11:497.

doi: $10.3389 /$ fendo.2020.00497

\section{Linear and Non-linear Correlations Between Serum Phosphate Level and Bone Mineral Density in Type 2 Diabetes}

\author{
Yinqiu Yang ${ }^{1 \dagger}$, Guangwang Liu ${ }^{2 \dagger}$, Yao Zhang ${ }^{1,3}$, Guiping $X u^{1,4}$, Xilu Yi ${ }^{1,5}$, Jing Liang ${ }^{1}$, \\ Chenhe Zhao ${ }^{1}$, Jun Liang ${ }^{6}$, Chao $\mathrm{Ma}^{2}$, Yangli Ye ${ }^{1}$, Mingxiang $\mathrm{Yu}^{1 *}$ and Xinhua $\mathrm{Qu}{ }^{7 *}$ \\ ${ }^{1}$ Department of Endocrinology, Zhongshan Hospital, Fudan University, Shanghai, China, ${ }^{2}$ Department of Orthopaedics, \\ Xuzhou Central Hospital, Xuzhou Clinical School of Xuzhou Medical University, The Affiliated Xuzhou Hospital of Medical \\ College of Southeast University, Xuzhou Clinical Medical College of Nanjing University of Chinese Medicine, Xuzhou, China, \\ ${ }^{3}$ Department of Infectious Disease, Zhongshan Hospital, Fudan University, Shanghai, China, ${ }^{4}$ VIP Clinical Department, Fujian \\ Provincial Hospital, Fuzhou, China, ${ }^{5}$ Department of Endocrinology, Songjiang Central Hospital, Shanghai, China, \\ ${ }^{6}$ Department of Endocrinology, Xuzhou Central Hospital, Xuzhou Clinical School of Xuzhou Medical University, The Affiliated \\ Xuzhou Hospital of Medical College of Southeast University, Xuzhou Clinical Medical College of Naniing University of Chinese \\ Medicine, Xuzhou, China, ${ }^{7}$ Department of Bone and Joint Surgery, Renji Hospital, Shanghai Jiaotong University School of \\ Medicine, Shanghai, China
}

Introduction: Serum phosphate plays an important role in bone mineralization and might be a risk factor for many bone diseases. Patients with T2D usually have low serum phosphate level due to diet control, osmotic diuresis, and insulin stimulation. Current studies have discussed the linear association between serum phosphate and bone mineral density (BMD).

Objective: We aimed to analyze both the linear and non-linear correlations between serum phosphate and BMD in patients with type 2 diabetes (T2D).

Methods: We included 1,469 patients with T2D and obtained their basic information, laboratory measurements, and BMD data. Multivariate adjusted linear regression was used to analyze the linear associations, and we applied a two-piecewise linear regression model using a smoothing function to examine the non-linear association.

Results: No linear correlation was found between serum phosphate and BMD in patients with T2D. In women with T2D, we found a non-linear correlation between serum phosphate level and femur neck or total hip BMD. When serum phosphate was $<1.3$ $\mathrm{mmol} / \mathrm{L}$, it was positively associated with femur neck and total hip BMD, whereas when phosphate was $>1.3 \mathrm{mmol} / \mathrm{L}$, it was negatively associated with femur neck BMD.

Conclusions: In men with T2D, serum phosphate level was not associated with BMD. However, in women with T2D, we found a non-linear correlation between serum phosphate and femur neck or total hip BMD.

Keywords: serum phosphate, bone mineral density, type 2 diabetes, bone metabolism, fracture risk 


\section{INTRODUCTION}

Over the past few years, the incidence of diabetes has increased significantly in both developed and developing countries. According to the 2018 study, the global prevalence of diabetes is about $8.8 \%(1)$. Diabetes as a chronic disease, is manifested by typical clinical polydipsia, polyuria, and more food and weight loss, and could cause many serious short-term and longterm complications, including myocardial infarction, diabetic retinopathy, neuropathy, making diabetes a growing global health concern (2). Studies have shown that in patients with type 2 diabetes (T2D), the incidence of osteoporosis and the risk of fracture are significantly increased and usually have poor prognosis $(3,4)$, which leads to an increased burden to the family and society. Finding the risk factors of osteoporosis in T2D is of great significance. Bone mineral density could be affected by various factors. Studies have already proved that factors including age, body mass index (BMI), smoking, alcohol use, diabetes, other endocrine diseases like parathyroid diseases, kidney function, liver function, blood pressure, Vitamin $\mathrm{D}$, and growth factors levels are all the risk factors of low bone mass (5-7).

A study has shown that the serum phosphorus level was significantly lower in patients with T2D than in controls, which suggested a disorder of phosphorus metabolism in T2D (8). We speculate that there might be a correlation between this phosphorus metabolism disorder and bone metabolism disorder. In mammalian systems, phosphorus is a key element in multiple physiological processes, especially in bone mineralization (9). Phosphorus is mainly absorbed from the intestines (10) and exists in the form of inorganic phosphate (P-3) in living organisms. Serum phosphate concentration increases within hours after absorption (11). Eighty-five percent of phosphate is stored as hydroxyapatite in bones and teeth, whereas only 14 and $1 \%$ remain in intracellular and extracellular fluids, respectively, (12).

Maintenance of phosphate homeostasis is centered on the regulation of phosphate handling by the bones, intestines, and kidneys. In the skeleton, phosphate complexes with calcium and is stored as hydroxyapatite crystals, and plays an important role in bone matrix mineralization (13). The rate of bone remodeling is partly dependent on the concentration of phosphate and calcium. Without these elements, mineralization will be impaired, leading to the formation of poorly mineralized bone, which results in osteomalacia or rickets (9).

Serum phosphate seems to play a key role in bone mineralization and formation, and phosphate disorder might cause many bone diseases and fractures. In recent years, the correlation between serum phosphate level and bone mineral density (BMD) in different populations has been widely studied. A cohort study in 2017 found that serum phosphate was negatively associated with BMD in postmenopausal women, but not in men (14). Another study based on two population-based cohorts reported a negative relationship between phosphate and lumbar spine BMD in men and no association between phosphate and femur neck BMD in both sexes (15). The inverse association between serum phosphate and total body BMD in men was also found by Clarke et al. (16).
Among all the studies, only one Chinese retrospective study was conducted in patients with $\mathrm{T} 2 \mathrm{D}$, which showed that BMD was not correlated with serum phosphate (17). Furthermore, almost all related studies only discussed the linear correlation. Therefore, we conducted our study based on the population with T2D and aimed to clarify both the linear and non-linear correlations between serum phosphate and total lumbar, femur neck, and total hip BMDs. The null hypothesis of our study is that serum phosphate level is irrelevant to BMD.

\section{MATERIALS AND METHODS Study Population}

This study was a retrospective and cross-sectional study based on consecutively selected T2D patients who hospitalized in the Endocrinology Department of Zhongshan Hospital between October 2009 and January 2013. All participants were older than 18 years with definite T2D diagnosis based on the American Diabetes Association's Standards of Medical Care in Diabetes (18). They all denied recent intake of calcium tablets, diphosphonate, vitamin $\mathrm{D}$, or other drugs that might influence bone metabolism or serum phosphate level. We excluded those who had heart failure, renal, or hepatic insufficiency, serious cardiovascular disease, malignant tumor, or other endocrine diseases. Disabled patients or those bed-ridden for a long period were also excluded. Our study finally included 1,469 patients with T2D (866 men and 603 women). We used individual questionnaires to determine the medical and personal history and other relevant information of each participant. Our study was approved by the ethics committee of Zhongshan Hospital, Fudan University (Approval No. B2017-172R).

\section{Basic Information}

All participants voluntarily provided their personal and family information, and they all received a comprehensive physical examination in our hospital. We measured the blood pressure of each participant after a 30 -min rest and in sitting position. We measured the height and weight of each patient in the morning after urination and before breakfast. The measurements were conducted more than once, and the average value was used. The duration of diabetes was calculated in the unit of years from the initial T2D diagnosis to the time we collected their basic information and obtained blood samples. The treatment of diabetes included oral hypoglycemic drugs, insulin injection, both hypoglycemic drugs and insulin, or none. Smoking history, alcohol intake history, disease history, and family history were defined as never or ever.

\section{Laboratory Measurements}

During hospitalization, the serum samples of each participant were collected at 6 A.M after overnight fasting. The assays of these samples were performed within $4 \mathrm{~h}$ at room temperature. Serum phosphate concentration was tested by molybdenum blue method. The test sensitivity was $0.01 \mathrm{mmol} / \mathrm{L}$ and the coefficient of variation is $<4.0 \%$. 
TABLE 1 | Patient characteristics, stratified by sex.

\begin{tabular}{|c|c|c|c|c|}
\hline & $\begin{array}{c}\text { Total patients } \\
(n=1,469) \\
\text { (Mean } \pm \text { SD or } N \%)\end{array}$ & $\begin{array}{c}\text { Male patients } \\
\quad(n=866) \\
\text { (Mean } \pm \text { SD or } N \%)\end{array}$ & $\begin{array}{c}\text { Female patients } \\
(n=603) \\
\text { (Mean } \pm \text { SD or } N \%)\end{array}$ & $P$-value \\
\hline Diabetic duration (years) & $7.142 \pm 6.886$ & $6.089 \pm 6.413$ & $8.655 \pm 7.255$ & $<0.001$ \\
\hline Systolic blood pressure & $130.720 \pm 16.314$ & $129.521 \pm 15.565$ & $132.441 \pm 17.200$ & $<0.001$ \\
\hline Diastolic blood pressure & $80.842 \pm 9.351$ & $81.275 \pm 9.279$ & $80.221 \pm 9.427$ & 0.033 \\
\hline $\mathrm{FBG}, \mathrm{mmol} / \mathrm{l}$ & $8.699 \pm 3.051$ & $8.721 \pm 2.978$ & $8.667 \pm 3.156$ & 0.738 \\
\hline $\mathrm{HbA} 1 \mathrm{C}, \%$ & $9.325 \pm 2.364$ & $9.515 \pm 2.408$ & $9.055 \pm 2.275$ & $<0.001$ \\
\hline hsCRP, mg/l & $24.920 \pm 8.830$ & $4.319 \pm 9.458$ & $4.904 \pm 11.201$ & 0.296 \\
\hline $\mathrm{TC}, \mathrm{mmol} / \mathrm{l}$ & $4.579 \pm 1.082$ & $4.573 \pm 1.082$ & $4.588 \pm 1.084$ & 0.793 \\
\hline $\mathrm{TG}, \mathrm{mmol} / \mathrm{l}$ & $1.908 \pm 1.344$ & $1.957 \pm 1.409$ & $1.838 \pm 1.241$ & 0.097 \\
\hline eGFR (MDRD), $\mathrm{ml} / \mathrm{min} / 1.73 \mathrm{~m}^{2}$ & $104.395 \pm 39.293$ & $93.270 \pm 33.813$ & $120.366 \pm 41.090$ & $<0.001$ \\
\hline $\mathrm{K}, \mathrm{mmol} / \mathrm{l}$ & $3.972 \pm 0.383$ & $3.976 \pm 0.383$ & $3.965 \pm 0.383$ & 0.587 \\
\hline $\mathrm{Na}, \mathrm{mmol} / \mathrm{l}$ & $141.541 \pm 3.216$ & $141.587 \pm 3.206$ & $141.476 \pm 3.232$ & 0.516 \\
\hline $\mathrm{Ca}, \mathrm{mmol} / \mathrm{l}$ & $2.226 \pm 0.113$ & $2.227 \pm 0.116$ & $2.225 \pm 0.109$ & 0.681 \\
\hline $\mathrm{Mg}, \mathrm{mmol} / \mathrm{l}$ & $0.854 \pm 0.085$ & $0.853 \pm 0.084$ & $0.855 \pm 0.088$ & 0.626 \\
\hline $\mathrm{TB}, \mathrm{umol} / \mathrm{l}$ & $10.284 \pm 4.666$ & $10.241 \pm 4.508$ & $10.346 \pm 4.887$ & 0.673 \\
\hline $\mathrm{CB}, \mathrm{umol} / \mathrm{l}$ & $3.720 \pm 1.767$ & $3.707 \pm 1.772$ & $3.739 \pm 1.762$ & 0.732 \\
\hline $\mathrm{ALT}, \mathrm{U} / \mathrm{L}$ & $27.093 \pm 30.632$ & $27.498 \pm 29.295$ & $26.514 \pm 32.462$ & 0.546 \\
\hline AST, U/L & $23.658 \pm 20.519$ & $24.029 \pm 21.201$ & $23.126 \pm 19.508$ & 0.408 \\
\hline $\mathrm{PTH}, \mathrm{pg} / \mathrm{ml}$ & $36.468 \pm 14.573$ & $36.513 \pm 14.580$ & $36.401 \pm 14.577$ & 0.888 \\
\hline $25(\mathrm{OH}) \mathrm{D}, \mathrm{nmol} / \mathrm{L}$ & $34.834 \pm 17.058$ & $34.384 \pm 17.104$ & $35.496 \pm 16.982$ & 0.231 \\
\hline Oral medicine & $607(41.321 \%)$ & $352(40.647 \%)$ & $255(42.289 \%)$ & \\
\hline Insulin and OM & $255(17.359 \%)$ & $146(16.859 \%)$ & 109 (18.076\%) & \\
\hline Smoking & & & & $<0.001$ \\
\hline Never & $1,078(73.383 \%)$ & $488(56.351 \%)$ & $590(97.844 \%)$ & \\
\hline Current or ever & 391 (26.617\%) & $378(43.649 \%)$ & $13(2.156 \%)$ & \\
\hline Drinking & & & & $<0.001$ \\
\hline Never & $1,275(86.794 \%)$ & $679(78.406 \%)$ & $596(98.839 \%)$ & \\
\hline Current or ever & $194(13.206 \%)$ & $187(21.594 \%)$ & $7(1.161 \%)$ & \\
\hline Hypertension & & & & $<0.001$ \\
\hline No & $781(53.165 \%)$ & $502(57.968 \%)$ & 279 (46.269\%) & \\
\hline Yes & $688(46.835 \%)$ & 364 (42.032\%) & 324 (53.731\%) & \\
\hline Gout or hyperuricemia & & & & 0.895 \\
\hline No & $1,443(98.230 \%)$ & 851 (98.268\%) & 592 (98.176\%) & \\
\hline Yes & $26(1.770 \%)$ & $15(1.732 \%)$ & $11(1.824 \%)$ & \\
\hline Family history of diabetes & & & & 0.044 \\
\hline No & 878 (59.769\%) & $499(57.621 \%)$ & $379(62.852 \%)$ & \\
\hline Yes & 591 (40.231\%) & 367 (42.379\%) & 224 (37.148\%) & \\
\hline
\end{tabular}

Values are mean $\pm S D$ or $n(\%)$ unless otherwise specified. 
We also evaluated the blood cell counts, indexes of hepatic function, renal function, blood electrolytes, glucose, and lipid metabolism, and bone turnover markers, and others.

\section{BMD Measurement}

According to the International Society for Clinical Densitometry guidelines, we used dual-energy X-ray absorptiometry (HologicDiscovery, USA) to measure the BMD of each patient at three different sites: total lumbar, femur neck, and total hip.

\section{Statistical Analysis}

In men and women with $\mathrm{T} 2 \mathrm{D}$, both linear and non-linear correlations between serum phosphate level and BMD were analyzed separately. Chi-square test was used to analyze categorical variables, which were expressed as numbers and proportions. Continuous variables were expressed as mean and standard deviation (SD). One-way analysis of variance was used for normally distributed continuous variables, and KruskalWallis test was used for skewed continuous variables.

We used multivariate adjusted linear regression and $T$ test to analyze the linear associations between each SD in serum phosphate and BMD. We calculated the regression coefficient and corresponding 95\% confidence intervals (CI). Adjusted model I was adjusted for age, body mass index (BMI), treatment for diabetes mellitus (DM), alcohol intake, smoking, and hypertension. Adjusted model II was adjusted for age, BMI, hypertension, systolic blood pressure, diastolic blood pressure, diabetes duration (years), treatment for DM, smoking, alcohol intake, family history of DM, fasting blood glucose (FBG; mmol/L), BUN (mmol/L), Cr ( $\mu \mathrm{mol} / \mathrm{L})$, eGFR (MDRD; $\left.\mathrm{ml} / \mathrm{min} / 1.73 \mathrm{~m}^{2}\right), \mathrm{Ca}(\mathrm{mmol} / \mathrm{L}), \operatorname{ALT}(\mathrm{U} / \mathrm{L}), \operatorname{AST}(\mathrm{U} / \mathrm{L})$, ALP (U/L), 25(OH)D, mmol/L and PTH, pg/ml. A $P<0.05$ was considered statistically significant.
We further examined the non-linear association between serum phosphate level and BMD. A smoothing function and a piecewise-linear regression model were applied, and we also adjusted age; treatment of DM; diabetic duration(y); smoking; drinking; family history of DM; BMI; hypertension; systolic blood pressure; diastolic blood pressure; FBG, unit; $\mathrm{Cr}, \mu \mathrm{mol} / \mathrm{l}$; BUN, $\mathrm{mmol} / \mathrm{l}$; eGFR (MDRD), $\mathrm{mL} / \mathrm{min} / 1.73 \mathrm{~m}^{2}$; Ca, unit; ALT, U/L; AST, U/L; ALP, U/L; 25(OH)D, mmol/L, and PTH, pg/ml.

We used $\mathrm{R}$ packages (http://www.r-project.org) and Empower ${ }^{\circledR} \quad(R) \quad$ (www.empowerstats.com, X\&Y Solutions Inc., Boston, MA, USA) for the statistical analyses.

\section{RESULTS}

\section{Characteristics of Participants}

Our study finally included 1,469 patients with T2D (866 men and 603 women). Table 1 describes the distribution of relevant covariates. The mean age of all participants was 56.88 years, and the mean diabetes duration was 7.142 years. The mean BMD values were $0.966,0.758$, and $0.902 \mathrm{~g} / \mathrm{cm}^{2}$ at total lumbar, femur neck, and total hip, respectively. We separately described the characteristics of the patients by sex and found several differences between them. The average age of the women was higher than that of the men. Diabetes duration in women was longer, but the mean $\mathrm{HbA} 1 \mathrm{c}$ level was lower than that in men. The percentage of alcoholic drinkers and smokers in men was much higher than in women. More men have family history of diabetes, whereas more women have high blood pressure.

\section{Linear Association Between Serum Phosphate and BMD}

In the crude model, we found a positive association between serum phosphate and femur neck or total hip BMD in

TABLE 2 | Multivariate regression for effect of serum phosphate level on total lumbar, femur neck, and total hip BMD.

\begin{tabular}{|c|c|c|c|c|c|c|}
\hline \multirow{2}{*}{$\begin{array}{l}\text { Phosphate, } \\
\text { mmol/L per SD }\end{array}$} & \multicolumn{2}{|l|}{ Male patients } & \multicolumn{2}{|c|}{ Female patients } & \multicolumn{2}{|l|}{ Total patients } \\
\hline & $\beta(95 \% \mathrm{Cl})$ & $\boldsymbol{P}$ & $\beta(95 \% \mathrm{Cl})$ & $\boldsymbol{P}$ & $\beta(95 \% \mathrm{Cl})$ & $P$ \\
\hline \multicolumn{7}{|l|}{ Total lumbar BMD } \\
\hline Crude model & $0.0091(-0.0028,0.0211)$ & 0.135495 & $-0.0064(-0.0207,0.0079)$ & 0.383089 & $0.0024(-0.0067,0.0116)$ & 0.602469 \\
\hline Adjusted model I & $0.0082(-0.0038,0.0202)$ & 0.182079 & $-0.0066(-0.0213,0.0081)$ & 0.378642 & $0.0023(-0.0070,0.0116)$ & 0.630558 \\
\hline Adjusted model II & $0.0051(-0.0082,0.0184)$ & 0.453812 & $-0.0061(-0.0223,0.0102)$ & 0.465808 & $0.0011(-0.0091,0.0112)$ & 0.837072 \\
\hline \multicolumn{7}{|l|}{ Femur neck BMD } \\
\hline Crude model & $0.0102(0.0002,0.0203)$ & 0.045939 & $0.0064(-0.0053,0.0182)$ & 0.284658 & $0.0086(0.0010,0.0162)$ & 0.027405 \\
\hline Adjusted model I & $0.0101(-0.0001,0.0202)$ & 0.050471 & $0.0049(-0.0072,0.0170)$ & 0.424954 & $0.0080(0.0003,0.0158)$ & 0.041832 \\
\hline Adjusted model II & $0.0047(-0.0064,0.0158)$ & 0.408099 & $0.0037(-0.0095,0.0169)$ & 0.584031 & $0.0043(-0.0041,0.0128)$ & 0.312971 \\
\hline \multicolumn{7}{|l|}{ Total hip BMD } \\
\hline Crude model & $0.0108(0.0001,0.0215)$ & 0.047924 & $0.0069(-0.0057,0.0194)$ & 0.286173 & $0.0091(0.0010,0.0173)$ & 0.028562 \\
\hline Adjusted model I & $0.0103(-0.0005,0.0210)$ & 0.061224 & $0.0051(-0.0077,0.0180)$ & 0.434742 & $0.0082(-0.0001,0.0165)$ & 0.050309 \\
\hline Adjusted model II & $0.0046(-0.0071,0.0163)$ & 0.440900 & $0.0081(-0.0059,0.0221)$ & 0.259697 & $0.0059(-0.0030,0.0148)$ & 0.191654 \\
\hline
\end{tabular}

Non-adjusted model adjust for: None.

Adjust I model adjust for: Age; BMI; Treatment of DM; Drinking; Smoking; Hypertension.

Adjust Il model adjust for: age; treatment of DM; diabetic duration(y); smoking; drinking; family history of DM; BMl; hypertension; systolic blood pressure; diastolic blood pressure; FBG, $\mathrm{mmol} / \mathrm{L} ; \mathrm{Cr}, \mathrm{umol} / \mathrm{l} ; \mathrm{BUN}, \mathrm{mmol} / \mathrm{l} ; \mathrm{eGFR}(\mathrm{MDRD}), \mathrm{mL} / \mathrm{min} / 1.73 \mathrm{~m}^{2} ; \mathrm{Ca}, \mathrm{mmol} / \mathrm{L} ; A L T, \mathrm{U} / \mathrm{L} ; A S T, \mathrm{U} / \mathrm{L} ; A L P, \mathrm{U} / \mathrm{L} ; 25(\mathrm{OH}) \mathrm{D}, \mathrm{mmol} / \mathrm{L} ; \mathrm{PTH}, \mathrm{pg} / \mathrm{ml}$. 


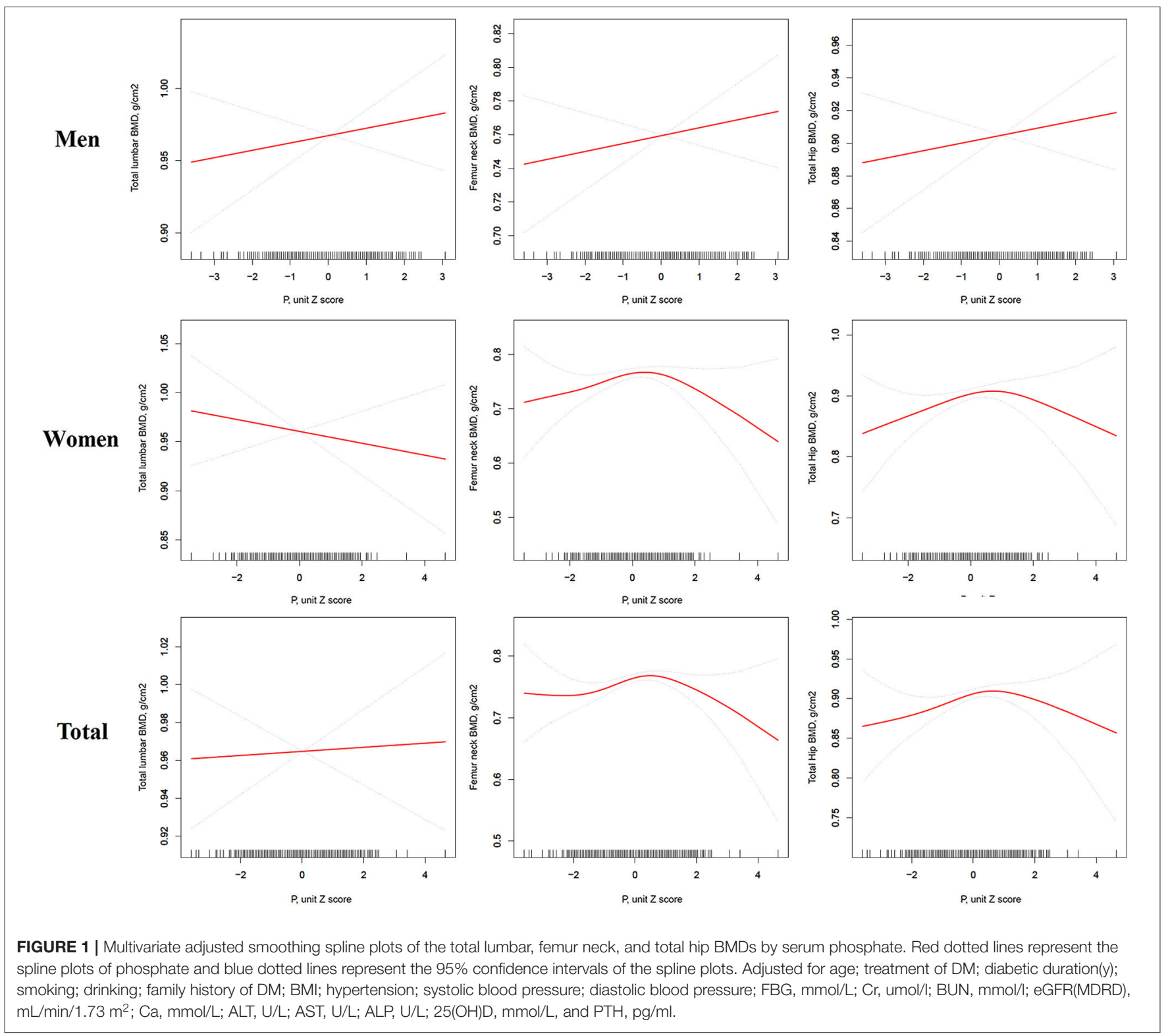

men with T2D (Table 2). With a 1-SD increase in serum phosphate level, femur neck BMD increased by $0.0102 \mathrm{~g} / \mathrm{cm}^{2}$ $(P=0.045939,95 \% \mathrm{CI}=0.0002-0.0203)$ and total hip BMD increased by $0.0108 \mathrm{~g} / \mathrm{cm}^{2}(P=0.047924,95 \% \mathrm{CI}=$ 0.0005-0.0210). However, after adjusting for age, BMI, blood pressure, diabetes duration, treatment for DM, smoking, alcohol intake, family history of DM, BUN, Cr, eGFR, FBG, Ca, ALT, AST, ALP, PTH, and $25(\mathrm{OH}) \mathrm{D}$, this positive correlation disappeared. No association between serum phosphate level and BMD was found in the crude or multivariate-adjusted models in women.

\section{Non-linear Association Between Serum Phosphate and BMD}

As shown in Figure 1, there might be some non-linear association between serum phosphate and BMD. We further applied a two-piecewise linear regression model (Table 3). Phosphate was found to be non-linearly correlated with femur neck and total hip BMD after multivariate adjustment in women. When phosphate was $<1.3 \mathrm{mmol} / \mathrm{L}$, serum phosphate was positively correlated with femur neck and total hip BMD. With a 1-SD increase in phosphate, femur neck BMD increased by $0.1259 \mathrm{~g} / \mathrm{cm}^{2}(P=0.0164,95 \% \mathrm{CI}=0.0235-0.2282)$ and total hip BMD increased by $0.1303 \mathrm{~g} / \mathrm{cm}^{2}(P=0.0199,95 \% \mathrm{CI}=$ $0.0211-0.2395)$. However, when phosphate was $>1.3 \mathrm{mmol} / \mathrm{L}$, we found a negative relationship between serum phosphate level and BMD at the femur neck and total hip. A 1-SD increase in phosphate was correlated with a $-0.1385 \mathrm{~g} / \mathrm{cm}^{2}(P=0.409,95 \%$ $\mathrm{CI}=-0.2708,-0.0016)$ decrease in femur neck BMD. However, in men with T2D, we found no non-linear correlation between serum phosphate level and total lumbar, femur neck, or total hip BMD. 
TABLE 3 | Non-linear association between serum phosphate level and BMD.

\begin{tabular}{|c|c|c|c|c|c|}
\hline \multirow{2}{*}{$\begin{array}{l}\text { Phosphate, } \\
\text { mmol/L per SD }\end{array}$} & \multirow{2}{*}{$\begin{array}{c}\text { Break } \\
\text { point (K) }\end{array}$} & \multicolumn{2}{|l|}{$<\mathbf{K}$} & \multicolumn{2}{|l|}{$>\mathbf{K}$} \\
\hline & & $\beta(95 \% \mathrm{Cl})$ & $p$ & $\beta(95 \% \mathrm{Cl})$ & $p$ \\
\hline \multicolumn{6}{|c|}{ Total lumbar BMD } \\
\hline Men & 1.3 & $0.0234(-0.0862,0.1331)$ & 0.6754 & $0.0273(-0.1027,0.1574)$ & 0.6807 \\
\hline Women & 1.3 & $-0.0393(-0.1667,0.0882)$ & 0.5462 & $-0.0163(-0.1808,0.1481)$ & 0.8456 \\
\hline Total & 1.3 & $-0.0009(-0.0832,0.0814)$ & 0.9829 & $0.0135(-0.0871,0.1142)$ & 0.7921 \\
\hline \multicolumn{6}{|l|}{ Femur neck BMD } \\
\hline Men & 1.3 & $0.0712(-0.0203,0.1628)$ & 0.1278 & $-0.0383(-0.1470,0.0705)$ & 0.4904 \\
\hline Women & 1.3 & $0.1259(0.0235,0.2282)$ & 0.0164 & $-0.1385(-0.2708,-0.0061)$ & 0.0409 \\
\hline Total & 1.3 & $0.0996(0.0318,0.1675)$ & 0.0041 & $-0.0835(-0.1665,-0.0004)$ & 0.0492 \\
\hline \multicolumn{6}{|l|}{ Total Hip BMD } \\
\hline Men & 1.3 & $0.0570(-0.0394,0.1534)$ & 0.2467 & $-0.0212(-0.1359,0.0934)$ & 0.7169 \\
\hline Women & 1.3 & $0.1303(0.0211,0.2395)$ & 0.0199 & $-0.0911(-0.2318,0.0496)$ & 0.2051 \\
\hline Total & 1.3 & $0.0972(0.0254,0.1690)$ & 0.0081 & $-0.0617(-0.1496,0.0263)$ & 0.1696 \\
\hline
\end{tabular}

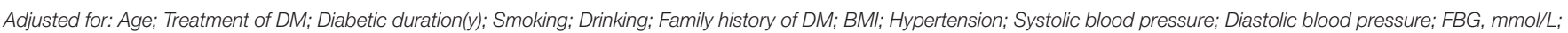
Cr, umol/l; BUN, mmol/l; eGFR(MDRD), $\mathrm{mL} / \mathrm{min} / 1.73 \mathrm{~m}^{2}$; Ca, mmol/L; ALT, U/L; AST, U/L; ALP, U/L; 25(OH)D, mmol/L; PTH, pg/ml.

\section{DISCUSSION/CONCLUSION}

In this cross-sectional study, no linear correlation between serum phosphate and BMD was found both in men and women with T2D. However, our study, for the first time, reported a nonlinear correlation between serum phosphate level and BMD. In women with $\mathrm{T} 2 \mathrm{D}$, we found a positive association between serum phosphate level and femur neck or total hip BMD with serum phosphate level $<1.3 \mathrm{mmol} / \mathrm{L}(P<0.05)$, but when the serum phosphate level was $>1.3 \mathrm{mmol} / \mathrm{L}$, the association became negative $(P<0.05)$. Thus, we can reject our null hypothesis. The normal range of blood phosphate is $0.90-1.34$ $\mathrm{mmol} / \mathrm{L}$, according to the test standard of Zhongshan Hospital. Combined with our findings, we can conclude that maintaining a relatively high blood phosphate level, but still within the normal range, might be beneficial to BMD, whereas a serum phosphate increase above the upper limit might have a negative effect on bone health. These conclusions suggested an ideal serum phosphate level $(1.3 \mathrm{mmol} / \mathrm{L})$ for women and help us set an appropriate target value for phosphate-reducing treatment. Moreover, women with too high or too low serum phosphate should monitor their bone mineral density more frequently in order to avoid osteoporotic fractures. However, in men with T2D, no non-linear correlation was found. The different results we found between men and women patients may be partly because of the different characteristics of these two groups. The average age of the women was higher and their diabetes duration was longer, but the mean $\mathrm{HbAlc}$ level was lower than that in men. Smoking, drinking history, hypertension history, and family history of diabetes also have remarkable differences in men and women.

Previous studies reported a negative relationship between serum phosphate level and BMD in both postmenopausal women and men $(14,16)$. However, another study based on two population-based cohorts, found that such a relationship varies at different sites. According to this study, serum was only inversely related to lumbar spine BMD in men, but no relationship with the femur neck was found in either sex. This study also discussed the association between serum phosphate and fracture risk and suggested a potential threshold of serum phosphate (men: $1.1 \mathrm{mmol} / \mathrm{L}$, women: $1.2 \mathrm{mmol} / \mathrm{L}$ ), above which fracture risk was increased (15), which is consistent with the results of our study. Both animal studies and clinical studies have found an association between phosphorus intake and BMD. Koyama et al. (19) found that a Pihigh diet significantly reduced both BMD and bone mass in wild-type mice. In human subjects, phosphorus intake exceeded the recommended dosage had an independent and adverse effect on bone mass (20). A Chinese study of T2D patients reported that BMD was not correlated with serum phosphate, but this study did not analyze the possible non-linear correlation (17).

Serum phosphate level may directly affect bone metabolism. Inorganic phosphate is an essential element for the development of osteogenic cells, not only because it is an integral component of apatite crystal but also because it can affect the production rate of the bone matrix. A positive relation exists between the plasma phosphate level and the rate of skeletal growth and/or mineralization. (21) Phosphate was also essential for osteoblast differentiation and extracellular matrix mineralization and played a vital part in the mutation of growth plate and the formation of secondary ossification center (22). Thus, phosphate deprivation interfers with normal osteoblastic function, which in turn influences the process of mineralization.

Animal studies reported that calcium alone is insufficient for bone development in growing rats. At equivalent levels of calcium supplementation, the calcium phosphate salts promoted significantly greater body weight gain, femur 
weight, tensile strength, bone ash, bone mineralization, bone density, calcium, and phosphorus deposition, and calcium utilization (23). According to animal studies, a high-phosphate diet disturbed phosphate homeostasis, increased bone resorption, and reduced bone mineralization $(19,24,25)$.

There might be many other mechanisms underlying the association between phosphate and BMD. High plasma phosphate concentrations can induce the synthesis of FGF-23 (26). FGF-23 binding to its receptor, participates in 1-hydroxylation of $25(\mathrm{OH}) \mathrm{D}$, and induce inactivation of $1,25(\mathrm{OH}) 2 \mathrm{D}$, which finally influence bone metabolism (27, 28). High-P diet-induced elevated PTH secretion leads to an increase in RANKL expression, which enhances osteoclastic bone resorption. Clinical studies also proved the positive association between PTH levels and bone turnover $(29,30)$ as well as the negative correlation between PTH and BMD (31). Thus, we adjusted 25(OH)D and PTH when analyzing the independent effect of serum phosphate on bone mineral density.

Compared with the general population, patients with T2D have a disorder of phosphorus metabolism. The low serum phosphate level in T2D may be due to diet control and inadequate phosphorus intake. Hyperglycemia leads to osmotic diuresis, and with the increase of urine sugar excretion, the excretion of calcium and phosphorus in the urine increases correspondingly, resulting in calcium and phosphorus loss (32, 33). Poor control of glucose level will lead to large doses of insulin and transfer of phosphorus from the extracellular compartment to the intracellular compartment, which finally leads to low serum phosphate (34). Meanwhile, low phosphorus can lead to increased insulin resistance and decreased insulin sensitivity $(35,36)$. As is known, the incidence of osteoporosis and the risk of fracture were significantly increased in T2D $(3,4)$. According to our study and other studies mentioned above, this increase may be partly explained by the disorder of phosphorus metabolism.

Our study, for the first time, reported a non-linear association between serum phosphate level and BMD. Studies proved that with a 1-SD decrease in BMD, the risk of vertebral and non-vertebral fractures increased $1.7-3$ times $(37,38)$, which suggested BMD is an extremely useful parameter for classifying persons who are susceptible to osteoporotic fractures (39). In combination of our findings, patients maintaining an appropriate blood phosphate level might have higher BMD and lower fracture risk, while patients with too high or too low serum phosphate will be more vulnerable to osteoporotic fractures. Besides, we conducted our study in a large sample size of Asians with T2D and we adjusted for various possible confounding factors for the accuracy and reliability of the conclusion. However, our study has some limitations. First, the blood samples were collected only once, which may have caused measurement errors. Second, we used dual-energy Xray absorptiometry to measure the $\mathrm{BMD}$ value, which cannot separately measure trabecular and cortical BMDs. Third, some key variables could not be further detected, because this study is cross-sectional, and this may have affected the selection of confounding factors. Some important confounding factors might have been neglected, such as dietary habits, phosphate, and other micronutrients intake, physical activity, previous fractures, FGF-23 level, and for women, the menopausal status and estrogen level, which might have also influenced our study results. Thirdly, this is a cross-sectional study, which means the causal relationship between serum phosphate levels and BMD cannot be proven. Lastly, our study only discussed the association between serum phosphate and BMD, and the association with other bone-related indicators, such as bone metabolic markers and fracture risks, still needs to be further explored in future articles.

In conclusion, in patients with $\mathrm{T} 2 \mathrm{D}$, serum phosphate level was not linearly correlated with BMD. However, in women with T2D, we found a non-linear correlation between serum phosphate and femur neck and total hip BMDs. When the phosphate level was $<1.3 \mathrm{mmol} / \mathrm{L}$, the serum phosphate level was positively associated with femur neck and total hip BMDs. However, when the phosphate level was $>1.3 \mathrm{mmol} / \mathrm{L}$, we found a negative correlation between serum phosphate level and BMD at femur neck.

\section{DATA AVAILABILITY STATEMENT}

The datasets generated for this study are available on request to the corresponding author.

\section{ETHICS STATEMENT}

The studies involving human participants were reviewed and approved by Zhongshan Hospital, Fudan University. The patients/participants provided their written informed consent to participate in this study.

\section{AUTHOR CONTRIBUTIONS}

$\mathrm{XQ}$ and MY designed the research. YYa, JiL, CZ, and XQ conducted the research. GX, XY, YZ, YYe, JiL, CZ, and MY provided the essential reagents or the essential materials. YYa, GL, and XQ analyzed the data and/or performed the statistical analyses. YYa and GL wrote the manuscript. GL, JuL, CM, XQ, and MY critically revised the manuscript. All authors contributed to the article and approved the submitted version.

\section{FUNDING}

This study was supported by the Project of Health Innovation Teams of Xuzhou (Grant No. XWCX201601), Xuzhou Plan of introducing a team of clinical medical experts (2019), Key Research \& Development Plan of Jiangsu Provincial Department of Science and Technology (Grant No. BE2016640), and Jiangsu Health Commission of Health (Grant Nos. H201673, LGY2018047, ZDRCC2016022, and QNRC2016392). 


\section{REFERENCES}

1. Weisman A, Fazli GS, Johns A, Booth GL. Evolving trends in the epidemiology, risk factors, and prevention of type 2 diabetes: a review. Can J Cardiol. (2018) 34:552-64. doi: 10.1016/j.cjca.2018.03.002

2. Solli O, Stavem K, Kristiansen IS. Health-related quality of life in diabetes: the associations of complications with EQ-5D scores. Health Qual Life Outcomes. (2010) 8:18. doi: 10.1186/1477-7525-8-18

3. Piscitelli P, Neglia C, Vigilanza A, Colao A. Diabetes and bone: biological and environmental factors. Curr Opin Endocrinol Diabetes Obes. (2015) 22:43945. doi: 10.1097/MED.0000000000000203

4. Rathmann W, Giani G. Global prevalence of diabetes: estimates for the year 2000 and projections for 2030. Diabetes Care. (2004) 27:25689. doi: $10.2337 /$ diacare.27.10.2568

5. Cosman F, de Beur SJ, LeBoff MS, Lewiecki EM, Tanner B, Randall S, et al. Clinician's guide to prevention and treatment of osteoporosis. Osteoporos Int. (2014) 25:2359-81. doi: 10.1007/s00198-014-2794-2

6. Heidari B, Muhammadi A, Javadian Y, Bijani A, Hosseini R, Babaei M. Associated factors of bone mineral density and osteoporosis in elderly males. Int J Endocrinol Metab. (2017) 15:e39662. doi: 10.5812/ijem.39662

7. Bijelic R, Milicevic S, Balaban J. Risk factors for osteoporosis in postmenopausal women. Med Arch. (2017) 71:258. doi: 10.5455/medarh.2017.71.25-28

8. Fang L, Li X. [Level of serum phosphorus and adult type 2 diabetes mellitus]. Zhong Nan Da Xue Xue Bao Yi Xue Ban. (2016) 41:5026. doi: 10.11817/j.issn.1672-7347.2016.05.009

9. Berndt TJ, Schiavi S, Kumar R. "Phosphatonins" and the regulation of phosphorus homeostasis. Am J Physiol Renal Physiol. (2005) 289:F117082. doi: 10.1152/ajprenal.00072.2005

10. Heaney RP. Phosphorus nutrition and the treatment of osteoporosis. Mayo Clin Proc. (2004) 79:91-7. doi: 10.4065/79.1.91

11. Kärkkäinen M, Lamberg-Allardt C. An acute intake of phosphate increases parathyroid hormone secretion and inhibits bone formation in young women. J Bone Miner Res. (1996) 11:1905-12. doi: 10.1002/jbmr.5650111211

12. Herman H, Dallemagne MJ. The main mineral constituent of bone and teeth. Arch Oral Biol. (1961) 5:137-44. doi: 10.1016/0003-9969(61)90007-3

13. Farrow EG, White KE. Recent advances in renal phosphate handling. Nat Rev Nephrol. (2010) 6:207-17. doi: 10.1038/nrneph.2010.17

14. Billington EO, Gamble GD, Bristow S, Reid IR. Serum phosphate is related to adiposity in healthy adults. Eur J Clin Invest. (2017) 47:48693. doi: 10.1111/eci.12769

15. Campos-Obando N, Koek W, Hooker ER, van der Eerden BC, Pols HA, Hofman A, et al. Serum phosphate is associated with fracture risk: the rotterdam study and MrOS. J Bone Miner Res. (2017) 32:118293. doi: 10.1002/jbmr.3094

16. Clarke L, Ebeling PR, Jones JD, Wahner HW, O'Fallon WM, Riggs BL, et al. Predictors of bone mineral density in aging healthy men varies by skeletal site. Calcif Tissue Int. (2002) 70:137-45. doi: 10.1007/s00223-001-1072-4

17. Zhang YX, Min SX. [Analysis of clinical factors correlating with osteoporosis in patients with type 2 diabetes mellitus]. Nan Fang Yi Ke Da Xue Xue Bao. (2016) 36:1546-9

18. American Diabetes Association. Standards of medical care in diabetes-2012. Diabetes Care. (2012) 35(Suppl. 1):S11-63. doi: 10.2337/dc12-s011

19. Koyama Y, Rittling SR, Tsuji K, Hino K, Salincarnboriboon R, Yano T, et al. Osteopontin deficiency suppresses high phosphate load-induced bone loss via specific modulation of osteoclasts. Endocrinology. (2006) 147:30409. doi: 10.1210/en.2005-0671

20. Metz JA, Anderson JJ, Gallagher PJ. Intakes of calcium, phosphorus, and protein, and physical-activity level are related to radial bone mass in young adult women. Am J Clin Nutr. (1993) 58:537-42. doi: 10.1093/ajcn/5 8.4.537

21. Bingham PJ, Raisz LG. Bone growth in organ culture: effects of phosphate and other nutrients on bone and cartilage. Calcif Tissue Res. (1974) 14:3148. doi: 10.1007/BF02060281

22. Zhang R, Lu Y, Ye L, Yuan B, Yu S, Qin C, et al. Unique roles of phosphorus in endochondral bone formation and osteocyte maturation. J Bone Miner Res. (2011) 26:1047-56. doi: 10.1002/jbmr.294
23. Shapiro R, Heaney RP. Co-dependence of calcium and phosphorus for growth and bone development under conditions of varying deficiency. Bone. (2003) 32:532-40. doi: 10.1016/S8756-3282(03)00060-7

24. Shah BG, Krishnarao GV, Draper HH. The relationship of $\mathrm{Ca}$ and $\mathrm{P}$ nutrition during adult life and osteoporosis in aged mice. J Nutr. (1967) 92:3042. doi: $10.1093 /$ jn/92.1.30

25. Amato D, Maravilla A, Montoya C, Gaja O, Revilla C, Guerra R, et al. Acute effects of soft drink intake on calcium and phosphate metabolism in immature and adult rats. Rev Invest Clin. (1998) 50:185-9.

26. Pekkinen M, Laine CM, Mäkitie R, Leinonen E, Lamberg-Allardt C, Viljakainen $\mathrm{H}$, et al. FGF23 gene variation and its association with phosphate homeostasis and bone mineral density in finnish children and adolescents. Bone. (2015) 71:124-30. doi: 10.1016/j.bone.2014.10.013

27. Quarles LD. Evidence for a bone-kidney axis regulating phosphate homeostasis. J Clin Invest. (2003) 112:642-6. doi: 10.1172/JCI200319687

28. Saito H, Kusano K, Kinosaki M, Ito H, Hirata M, Segawa H, et al. Human fibroblast growth factor-23 mutants suppress $\mathrm{Na}+$-dependent phosphate cotransport activity and 1alpha,25-dihydroxyvitamin D3 production. J Biol Chem. (2003) 278:2206-11. doi: 10.1074/jbc.M207872200

29. Chapuy MC, Schott AM, Garnero P, Hans D, Delmas PD, Meunier PJ. Healthy elderly French women living at home have secondary hyperparathyroidism and high bone turnover in winter. EPIDOS study group. J Clin Endocrinol Metab. (1996) 81:1129-33. doi: 10.1210/jcem.81.3.8772587

30. Ledger GA, Burritt MF, Kao PC, O’Fallon WM, Riggs BL, Khosla S. Role of parathyroid hormone in mediating nocturnal and age-related increases in bone resorption. J Clin Endocrinol Metab. (1995) 80:330410. doi: $10.1210 /$ jcem. 80.11 .7593443

31. Ooms ME, Roos JC, Bezemer PD, van der Vijgh WJ, Bouter LM, Lips P. Prevention of bone loss by vitamin D supplementation in elderly women: a randomized double-blind trial. J Clin Endocrinol Metab. (1995) 80:10528. doi: $10.1210 /$ jcem.80.4.7714065

32. Rana S, Morya RK, Malik A, Bhadada SK, Sachdeva N, Sharma G. A relationship between vitamin $\mathrm{D}$, parathyroid hormone, calcium levels and lactose intolerance in type 2 diabetic patients and healthy subjects. Clin Chim Acta. (2016) 462:174-7. doi: 10.1016/j.cca.2016.09.009

33. Osuka A, Matsuoka T, Idoguchi K. Is this the worst outcome of metabolic syndrome? Hypophosphatemia and resulting cardiac arrest during the treatment of diabetic ketoacidosis with hypertriglyceridemia. Intern Med. (2009) 48:1391-5. doi: 10.2169/internalmedicine.48.2236

34. Liamis G, Milionis HJ, Elisaf M. Medication-induced hypophosphatemia: a review. QJM. (2010) 103:449-59. doi: 10.1093/qjmed/hcq039

35. McCarty MF, DiNicolantonio JJ. Bioavailable dietary phosphate, a mediator of cardiovascular disease, may be decreased with plant-based diets, phosphate binders, niacin, and avoidance of phosphate additives. Nutrition. (2014) 30:739-47. doi: 10.1016/j.nut.2013.12.010

36. DeFronzo RA, Lang R. Hypophosphatemia and glucose intolerance: evidence for tissue insensitivity to insulin. N Engl J Med. (1980) 303:125963. doi: 10.1056/NEJM198011273032203

37. Johnell O, Kanis JA, Oden A, Johansson H, De Laet C, Delmas P, et al. Predictive value of BMD for hip and other fractures. J Bone Miner Res. (2005) 20:1185-94. doi: 10.1359/JBMR.050304

38. Ross PD. Osteoporosis. Frequency, consequences, and risk factors. Arch Intern Med. (1996) 156:1399-411. doi: 10.1001/archinte.1996.00440120051005

39. Eastell R. Commentary: bone density can be used to assess fracture risk. BMJ. (1999) 318:864-5.

Conflict of Interest: The authors declare that the research was conducted in the absence of any commercial or financial relationships that could be construed as a potential conflict of interest.

Copyright (C) 2020 Yang, Liu, Zhang, Xu, Yi, Liang, Zhao, Liang, Ma, Ye, Yu and Qu. This is an open-access article distributed under the terms of the Creative Commons Attribution License (CC BY). The use, distribution or reproduction in other forums is permitted, provided the original author(s) and the copyright owner(s) are credited and that the original publication in this journal is cited, in accordance with accepted academic practice. No use, distribution or reproduction is permitted which does not comply with these terms. 